\title{
MHD Partial Slip Flow and Heat Transfer of Nanofluids through a Porous Medium Over a Stretching Sheet with Convective Boundary Condition
}

\section{Yohannes Yirga*}

Department of Mathematics, College of Natural and Computational Sciences, Mekelle University, Ethiopia (*yohannesalbn@gmail.com).

\begin{abstract}
This paper investigates the boundary layer analysis for magnetohydrodynamic partial slip flow and heat transfer of nanofluids through porous media over a stretching sheet with convective boundary condition. Four types of nanoparticles, namely copper, alumina, copper oxide and titanium oxide in the ethylene glycol (50\%, i.e., $\operatorname{Pr}=29.86)$ and water (i.e., $\mathrm{Pr}=6.58)$ based fluids are studied. The governing highly nonlinear and coupled partial differential equations are solved numerically using fourth order Runge-Kutta method with shooting techniques. The velocity and temperature profiles are obtained and utilized to compute the skin friction coefficient and local Nusselt number for different values of the governing parameters viz. nanoparticle volume fraction parameter, magnetic field parameter, porosity parameter, velocity slip parameter and convective parameter. It is found that the velocity distribution of the nanofluids is a decreasing function of the magnetic parameter, porosity parameter, and velocity slip parameter. However, temperature of the nanofluids is an increasing function of magnetic field parameter, nanoparticle volume fraction parameter, porosity parameter, velocity slip parameter and convective parameter. The flow and heat transfer characteristics of the four nanofluids are compared. Moreover, comparison of the numerical results is made with previously published works for special cases and an excellent agreement is found.
\end{abstract}

Keywords: Magnetohydrodynamics, Partial Slip, Porous medium, Convective boundary, Nanofluid.

\section{INTRODUCTION}

The study of the boundary layer flow of an electrically conducting fluid has many applications in manufacturing and natural process which include cooling of electronic devices by fans, cooling of nuclear reactors during emergency shutdown, cooling of an infinite metallic plate in a cooling bath, textile and paper industries, glass-fiber production, manufacture of plastic and rubber sheets, the utilization of geothermal energy, the boundary layer control in the field of aerodynamics, food processing, plasma studies and in the flow of biological fluids .

Magnetohydrodynamics (MHD) is the study of the flow of electrically conducting fluids in a magnetic field. Many experimental and theoretical studies on conventional electrically conducting fluids indicate that magnetic field markedly changes their transport and heat transfer 
characteristics. Recently, the application of magnetohydrodynamics in the polymer industry and metallurgy has attracted the attention of many researchers. Several researches investigated the MHD flow (Jafar et al., 2011; Hamada et al., 2011; Beg et al., 2009; Anuar, 2010; Fang et al., 2009; Fang et al., 2010; Makinde, 2012).

The study of flows with partial slip is important in the micro-electromechanical systems. The flow in these systems deviates significantly from the traditional no-slip flow because of the micro-scale dimensions of these devices. Rarefied gas flows with slip boundary conditions are often encountered in the micro-scale devices and low-pressure situations (Wang, 2009; Fang et al., 2010).

Flows through porous media have several applications present in nature: flow in sand beds, petroleum reservoir rocks, slurries, sedimentation, etc. Many industrial applications involve the modeling of flow through porous media, such as filters, catalyst beds, and packing. Porous materials are also used in various engineering devices such as catalytic converters and fuel cells. The rate of cooling can be controlled if strips are drawn through a porous medium. The flow of fluids through a porous medium under different conditions was studied by Abel et al., (2010a); Abel et al., (2010b); Abel et al. (2010c); Attia et al. (2012); Attia (2007); Damseh and Duwairi (2008); Dash et al. (2008); Magyari and Postelnicu (2011); Hamad and Pop (2011).

The similarity solution for laminar thermal boundary layer over a flat plate with a convective surface boundary condition was first studied by Aziz (2009). He demonstrates that, a similarity solution is possible if the convective heat transfer associated with the hot fluid on the lower surface of the plate is proportional to $x^{-1 / 2}$. Aziz also investigated hydrodynamic and thermal slip flow boundary layer over a flat plate with constant heat flux (Aziz, 2010). Since then, different researches have extended to different aspects of the boundary layer flow (Ishak et al., 2011; Wubshet and Shankar, 2012; Makinde and Olanrewaju, 2010; Yao et al., 2011; Ishak, 2010; Markin and Pop, 2011; Yacob et al., 2011).

Fluid heating and cooling are important in many industries such as power, manufacturing, transportation, and electronics. Effective cooling techniques are greatly needed for cooling any sort of high-energy device. Common heat transfer fluids such as water, ethylene glycol, and engine oil have limited/poor heat transfer capabilities due to their low heat transfer properties. In contrast, metals have thermal conductivities up to three times higher than these fluids, so it is natural that it 
would be desired to combine the two substances to produce a heat transfer medium that behaves like a fluid, but has the thermal conductivity of a metal. A lot of experimental and theoretical researches have been done to improve the thermal conductivity of the natural fluids.

In 1993, during an investigation of new coolants and cooling technologies at Argonne national laboratory, Choi invented a new type of fluid called Nanofluid (Sarit et al., 2007). Nanofluids are fluids that contain small volumetric quantities of nanometer-sized particles, called nanoparticles. The nanoparticles used in nanofluids are typically made of metals ( $\mathrm{Al}, \mathrm{Cu}, \mathrm{Ag}, \mathrm{Au}$, $\mathrm{Fe})$, oxides $\left(\mathrm{Al}_{2} \mathrm{O}_{3}, \mathrm{CuO}, \mathrm{TiO}_{2}\right)$, metal carbides ( $\left.\mathrm{SiC}\right)$, nonmetals (graphite carbon nanotubes), nitrides (AlN, SiN) and others. Common base fluids include water, ethylene glycol and oil. Nanofluids commonly contain up to a 5\% volume fraction of nanoparticles to see effective heat transfer enhancements. Nanofluids are studied because of their heat transfer properties: they enhance the thermal conductivity and convective properties over the properties of the base fluid. Moreover, the presence of the nanoparticles enhance the electrical conductivity property of the nanofluids, hence are more susceptible to the influence of magnetic field than the conventional base fluids. The suspended metallic or nonmetallic nanoparticles change the transport properties and heat transfer characteristics of the base fluid. Nanofluids have enhanced thermo-physical properties such as thermal conductivity, thermal diffusivity, viscosity and convective heat transfer coefficients compared to those of base fluids. Typical thermal conductivity enhancements are in the range of $15-40 \%$ over the base fluid and heat transfer coefficient enhancements have been found up to $40 \%$ ( $\mathrm{Yu}$ et al., 2008). Thermo-physical properties of nanofluids have been enormously studied by various workers, such as Kang et al. (2006); Velagapudi et al. (2008); Rudyak et al. (2010) and others.

After the pioneer investigation of Choi (Yu et al., 2008) various thriving experimental and theoretical researches were undertaken to discover and understand the mechanisms of heat transfer in nanofluids. The knowledge of the physical mechanisms of heat transfer in nanofluids is of vital importance as it will enable the exploitation of their full heat transfer potential. Musuda et al. (1993) observed that the characteristic feature of nanofluid is thermal conductivity enhancement. This observation suggests the possibility of using nanofluids in advanced nuclear systems (Buongiorno and $\mathrm{Hu}, 2005$ ). A comprehensive survey of convective transport in nanofluids made by Buongiorno (2006) indicated that a satisfactory explanation for the abnormal increase of the 
thermal conductivity and viscosity is yet to be found. Buongiorno (2006) further focused on heat transfer enhancement observed in convective situations. Khan and Pop (2011) suggested a similar solution for the free convection boundary layer flow past a horizontal flat plate embedded in a porous medium filled with a nanofluid. Makinde and Aziz (2010) studied MHD mixed convection from a vertical plate embedded in a porous medium with a convective boundary condition. Wubshet et al. (2013) have studied MHD stagnation point flow and heat transfer due to nanofluid towards a stretching sheet, while Turkyilmazoglua and Pop (2013) have studied heat and mass transfer of unsteady natural convection flow of some nanofluids past a vertical infinite flat plate with radiation effect and Xua et al. (2013) have investigated flow and heat transfer in a nanoliquid film over an unsteady stretching surface.

The interaction of magnetic field with nanofluids have several potential applications and may be used to deal with problems such as cooling of nuclear reactors by liquid sodium and induction flow meter which depends on the potential difference in the fluid in the direction perpendicular to the motion and to the magnetic field (Ganesan and Palani, 2004). During chemotherapy failure to provide localized drug targeting, results in an increase of toxic effects on neighboring organs and tissues, this is precisely done by magnetic drug targeting. This technology is based on binding established anticancer drugs with magnetic nanoparticles (ferrofluids) that concentrate the drug in the area of interest (tumor site) by means of magnetic fields. The above literature review reveals that most of the previous investigations are restricted to boundary layer flow and heat transfer in Newtonian fluids. However, due to the increasing importance of nanofluids in recent years a great attention has been given to the study of convective transport of nanofluids together with the magnetohydrodynamics. In addition to this, majority of the studies are carried without taking the effects of the solid volume fraction, nature of nanoparticle and base fluid into account. Since the flow and heat transfer characteristics of one type of nanofluid is different from the other, depending on the characteristics of the type of nanoparticle, solid volume fraction and the base fluid being used in the nanofluid. Moreover, the flow of nanofluids through porous media with a convective boundary condition has been given less attention. Therefore, the aim of the present paper is to study the combined effect of magnetic field, partial slip and porosity media on the flow and heat transfer of different nanofluids over a stretching sheet with convective boundary condition. The combined effect of all the above mentioned parameters has not been 
reported so far in the literature. Hence the study would help to supplement this unrevealed gap in the area.

The governing highly nonlinear partial differential equation of momentum and energy fields have been simplified by using a suitable similarity transformations and then solved numerically using fourth order Runge-Kutta method with shooting techniques. The effects of the governing parameters on the velocity and temperature have been discussed and presented in tables and graphs.

\section{MATHEMATICAL FORMULATION}

In this paper a steady two-dimensional laminar boundary layer flow of nanofluids is considered over a stretching sheet with a linear velocity $u_{w}(x)=a x$, where, $a$ is a constant and $x$ is the coordinate measured along the stretching surface and the flow takes place at $y \geq 0$, where $y$ is the coordinate measured normal to the stretching surface. Two equal and opposite forces are applied along the $\mathrm{x}$-axis so that the sheet is stretched keeping the origin fixed. The fluid is electrically conducting under the influence of an applied magnetic field $B_{0}(x)$ normal to the stretching surface. Since the magnetic Reynolds number is very small for most fluids used in industrial applications it is assumed that the induced magnetic field is negligible in comparison to the applied magnetic field. The fluid is water and ethylene glycol based nanofluid containing four different types of nanoparticles; namely copper, alumina, copper oxide and titanium oxide. It is assumed that the base fluids and the nanoparticles are in thermal equilibrium and no slip occurs between them. The thermo-physical properties of the base fluids and nanoparticles are given in table 1 (Oztop and Abu-Nada, 2008).

Table 1. Thermo-physical properties of water and base fluids and nanoparticles.

\begin{tabular}{|cllllll|}
\hline Physical properties & Water & Ethylene glycol & $\mathbf{C u}$ & $\mathbf{C u O}$ & $\mathbf{A l}_{\mathbf{2}} \mathbf{O}_{3}$ & $\mathbf{T i O}_{\mathbf{2}}$ \\
\hline $\boldsymbol{\rho}\left(\mathbf{K g} / \mathbf{m}^{3}\right)$ & 997.1 & 1056.1 & 8933 & 6320 & 3970 & 4250 \\
\hline $\mathbf{c}_{\mathbf{p}}(\mathbf{J} / \mathbf{K g K})$ & 4179 & 3287.5 & 385 & 531.8 & 765 & 686.2 \\
\hline $\mathbf{k}(\mathbf{W} / \mathbf{m K})$ & 0.613 & 0.426 & 400 & 76.5 & 40 & 8.954 \\
\hline
\end{tabular}

It is also assumed that, the bottom surface of the sheet is heated by convection from a hot fluid of temperature $T_{f}$ with a heat transfer coefficient $h$. Under the usual boundary layer approximations, (C) CNCS, Mekelle University 
the continuity, momentum and energy equations are given by:

$$
\begin{aligned}
& \frac{\partial u}{\partial x}+\frac{\partial u}{\partial y}=0 \\
& u \frac{\partial u}{\partial x}+v \frac{\partial u}{\partial y}=\frac{\mu_{n f}}{\rho_{n f}} \frac{\partial^{2} u}{\partial y^{2}}-\frac{\sigma B_{0}^{2}(x)}{\rho_{n f}} u-\frac{v_{n f}}{K_{0}} u \\
& u \frac{\partial T}{\partial x}+v \frac{\partial T}{\partial y}=\alpha_{n f} \frac{\partial^{2} T}{\partial y^{2}} .
\end{aligned}
$$

Subjected to the boundary condition

$$
u=u_{w}+A \frac{d u}{d y}, v=0,-k \frac{d T}{d y}=h\left(T_{f}-T\right) \text { at } y=0 u \rightarrow 0, T \rightarrow T_{\infty} \text {, as } y \rightarrow \infty
$$

Where, $u$ and $v$ are the velocity components in the $\mathrm{x}$ and $\mathrm{y}$ directions, respectively.

$T$ is the temperature of the nanofluid

$T_{\infty}$ is the temperature of the nanofluid far away from the sheet

$B_{0}$ is the uniform magnetic field strength

$\sigma$ is the electrical conductivity

$K_{0}$ is the permeability of the porous medium

$A$ is the velocity slip factor

$h$ is a heat transfer coefficient

$k$ is the thermal conductivity

$T_{f}$ is the convective temperature over the top surface of the plate.

$\mu_{n f}$ is the dynamic viscosity

$\rho_{n f}$ is density, and

$\alpha_{n f}$ is the thermal diffusivity of the nanofluid

(as given in Tiwari and Das (2007); and Ahmad et al., (2011))

$$
\begin{gathered}
v_{f}=\frac{\mu_{f}}{\rho_{f}}, \rho_{n f}=(1-\phi) \rho_{f}+\phi \rho_{s}, \quad \alpha_{n f}=\frac{k_{n f}}{\left(\rho c_{p}\right)_{n f}}, \mu_{n f}=\frac{\mu_{f}}{(1-\phi)^{2.5}} \\
\left(\rho c_{p}\right)_{n f}=(1-\phi)\left(\rho c_{p}\right)_{f}+\phi\left(\rho c_{p}\right)_{s}, \frac{k_{n f}}{k_{f}}=\frac{\left(k_{s}+2 k_{f}\right)-2 \phi\left(k_{f}-k_{s}\right)}{\left(k_{s}+2 k_{f}\right)+\phi\left(k_{f}-k_{s}\right)}
\end{gathered}
$$

In which $v_{f}, \mu_{f}, \rho_{f}$, and $k_{f}$ are the kinematic viscosity, dynamic viscosity, density, and thermal conductivity of the base fluid respectively; $\rho_{s}, k_{s}$, and $\left(\rho c_{p}\right)_{s}$ are the density, thermal conductivity, and heat capacitance of the nanoparticle respectively; $\phi$ is the solid volume fraction of nanoparticles; and $k_{n f}$ is thermal conductivity of the nanofluid.

To simplify the mathematical analysis of our study we introduce the following similarity transformations 


$$
\eta=y\left(\frac{a}{v_{f}}\right)^{\frac{1}{2}}, u=a f^{\prime}(\eta), v=-\left(a v_{f}\right)^{\frac{1}{2}} f(\eta), \theta(\eta)=\frac{T-T_{\infty}}{T_{f}-T_{\infty}}
$$

Making use of equation (6), the continuity equation (1) is automatically satisfied and equations. (2), (3) and the boundary condition (4) reduce to

$$
\begin{array}{r}
f^{\prime \prime \prime}+\phi_{1}\left[f f^{\prime \prime}-f^{2}-\frac{M}{\phi_{2}} f^{\prime}\right]-K_{1} f^{\prime}=0, \\
\theta^{\prime \prime}+\phi_{3} \operatorname{Pr}\left(\frac{k_{f}}{k_{n f}}\right) f \theta^{\prime}=0,
\end{array}
$$

With boundary conditions

$f(0)=0, f^{\prime}(0)=1+\gamma f^{\prime \prime}(0), \theta(0)=-h_{c}(1-\theta(0)), \quad f^{\prime}(\eta) \rightarrow 0, \theta(\eta) \rightarrow 0$, as $\eta \rightarrow \infty$

Where, $f(\eta)$ and $\theta(\eta)$ are the dimensionless velocity and temperature, respectively. Primes denote differentiation with respect to the similarity variable $\eta . h_{c}=\frac{h}{k} \sqrt{\frac{v_{f}}{a}}$ is convective parameter, $\quad M=\frac{\sigma B_{0}^{2}}{a \rho_{f}}, K_{1}=\frac{v_{f}}{a K_{0}}, \operatorname{Pr}=\frac{v_{f}\left(\rho c_{p}\right)_{f}}{k_{f}}$, and $\gamma=A \sqrt{\frac{a}{v_{f}}} \quad$ represent $\quad$ the magnetic parameter, porosity parameter, Prandtl number and the velocity slip parameter, respectively and $\phi_{1}, \phi_{2}$, and $\phi_{3}$ that depend on the nanoparticle volume fraction $\phi$ are given by

$$
\phi_{1}=(1-\phi)^{2}\left(1-\phi+\phi \frac{\rho_{s}}{\rho_{f}}\right), \phi_{2}=1-\phi+\phi \frac{\rho_{s}}{\rho_{f}}, \phi_{3}=1-\phi+\phi \frac{\left(\rho c_{p}\right)_{s}}{\left(\rho c_{p}\right)_{f}}
$$

The physical quantities of interest in this problem are the local skin friction coefficient $C_{f}$ and the Nusselt number $N u_{x}$ which represents the rate of heat transfer at the surface of the plate, which are defined as:

$$
C_{f}=\frac{2 \tau_{w}}{\rho_{f} u_{w}}, N u_{x}=\frac{x q_{w}}{k_{f}\left(T_{f}-T_{\infty}\right)}
$$

Where, $\tau_{w}$ is the skin friction and $q_{w}$ is the heat flux through the plate, which are given by

$$
\tau_{w}=\mu_{n f}\left(\frac{\partial u}{\partial y}\right)_{y=0}, q_{w}=k_{n f}\left(\frac{\partial T}{\partial y}\right)_{y=0}
$$

Making use of equations (5) and (6) in (11), the dimensionless skin friction coefficient and wall heat transfer rates are obtained as

$$
C_{f}(1-\phi)^{2.5} \sqrt{R e_{x}}=-f^{\prime \prime}(0), \frac{N x_{x}}{\sqrt{R e_{x}}} \frac{k_{f}}{k_{n f}}=-\theta^{\prime}(0)
$$

Where, $R e_{x}=\frac{x u_{w}}{v_{f}}$ is the local Reynolds number. 


\section{NUMERICAL METHOD}

The nonlinear boundary value problem represented by equations (7) to (9) is solved numerically using the fourth order Runge-Kutta method with shooting technique. In solving the system of nonlinear ordinary differential equations (7) and (8) together with the boundary condition (9) using the Runge-Kutta method, making an initial guess for the values of $f^{\prime \prime}(0)$ and $\theta^{\prime}(0)$ to initiate the shooting process is very important. The success of the scheme depends greatly on how much good is this guess to give the most accurate solution. The numerical solutions are obtained for several values of the governing parameters, viz. nanoparticle volume fraction parameter, magnetic field parameter, porosity parameter, velocity slip parameter and convective parameter. In this study a uniform grid of size $\Delta \eta=0.01$ is chosen to satisfy the convergence criteria of $10^{-6}$. The maximum value of $\eta_{\infty}$ was found to each iteration loop by $\eta_{\infty}=\eta_{\infty}+\Delta \eta$. The maximum value of $\eta_{\infty}$ to each group of parameter is determined when the value of the unknown boundary conditions at $\eta=0$ is not changed to successful loop with error less than $10^{-6}$.

\section{RESULTS AND DISCUSSION}

Heat transfer in MHD flow of nanofluids through a porous medium due to a stretching sheet with partial slip and convective boundary condition is studied considering four different types of nanoparticles namely, copper, copper oxide, alumina and titanium oxide, with water and ethylene glycol $(50 \%)$ as the base fluids (i.e. with a constant Prandtl numbers $\operatorname{Pr}=6.58$ and $\operatorname{Pr}=29.86$, respectively). The thermo-physical properties of the nanofluids were assumed to be functions of the nanoparticle volume fraction. The transformed nonlinear equations (7) and (8) subjected to the boundary condition (9) are solved numerically using Runge-Kutta method with shooting technique. The velocity and temperature profiles are obtained and utilized to compute the skinfriction coefficient and the local Nusselt number in equation (13). The numerical results for different values of the governing parameters viz. nanoparticle volume fraction parameter $\phi$, magnetic field parameter $M$, porosity parameter $K_{1}$, velocity slip parameter $\gamma$ and convective parameter $h_{c}$ are presented in graphs. In the absence of nanoparticles, to validate the accuracy of our results a comparison has been made with previously reported works in the literature. The comparisons are found to be in excellent agreement (see Tables 2 and 3).

The skin friction coefficients for different values of the magnetic parameter $M$ are given in 
table 2. It can be seen from table 2 that the magnitude of $f^{\prime \prime}(0)$ increases with an increase in $M$. Increasing values of $M$ resulted in a considerable opposition to the flow due to a Lorenz drag force. Moreover, comparison of the results with the solution as per Hayat et al. (2009) obtained from the closed form analytic solution of equation (7) subjected to the boundary condition (equation 9) given in equation (14) indicate an excellent agreement.

$$
f(\eta)=\frac{1-e^{-\sqrt{1+M} \eta}}{\sqrt{1+M}}, f^{\prime \prime}(0)=-\sqrt{1+M}
$$

Table 2. Comparison of the skin friction coefficient $-\mathrm{f}^{\prime \prime}(0)$ for different values of $\mathrm{M}$, when $\phi=$ $\mathrm{K}_{1}=\gamma=0, \operatorname{Pr}=6.2, \mathrm{~h}_{\mathrm{c}}=1000$.

\begin{tabular}{|lllllll|}
\hline $\boldsymbol{M}$ & $\mathbf{0}$ & $\mathbf{1}$ & $\mathbf{5}$ & $\mathbf{1 0}$ & $\mathbf{5 0}$ & $\mathbf{1 0 0}$ \\
\hline Exact [45] & 1 & 1.41421 & 2.44948 & 3.31662 & 7.14142 & 10.04987 \\
\hline Present & 1 & 1.41421 & 2.44949 & 3.31662 & 7.14142 & 10.04987 \\
\hline
\end{tabular}

Table 3. Comparison of the wall heat transfer rate $-\theta^{\prime}(0)$ for various values of $\phi$ and $\operatorname{Pr}$, when $\mathrm{M}=\mathrm{K}_{1}=\gamma=0, \mathrm{~h}_{\mathrm{c}}=1000$.

\begin{tabular}{|cllll|}
\hline $\boldsymbol{\phi}$ & $\boldsymbol{P r}$ & Numerical [46] & Analytic[47] & Present study \\
\hline 0.0 & 0.72 & 0.4631 & 0.463145 & 0.463145 \\
\hline & 1 & 0.5820 & 0.581977 & 0.581977 \\
\hline & 3 & 1.1652 & 1.165246 & 1.165249 \\
\hline & 10 & 2.3080 & 2.308004 & 2.308060 \\
\hline 0.1 & 6.2 & & & 1.468153 \\
\hline 0.2 & & & & 1.232378 \\
\hline
\end{tabular}

The local Nusselt number, which describe the rate of heat transfer coefficients are given in table 3 for different values of the Prandtl number $\operatorname{Pr}$ and the solid volume fraction parameter $\phi$. It can be observed that the rate of heat transfer at the surface increases with increasing values of $\mathrm{Pr}$ and decreases with increasing $\phi$. The present results showed good agreement with the earlier numerical results by Grubka and Bobba (1985), and the analytic solution given by Abramowitz and Stegun (1965) in terms of Kummer's functions, as given below:

$\theta(\eta)=e^{-P r \eta} \frac{M\left(P r, P r+1,-P r e^{-\eta}\right)}{M(P r, P r+1,-P r)}, \theta^{\prime}(0)=-P r+\frac{P r^{2}}{P r+1} \frac{M\left(P r, P r+1,-P r e^{-\eta}\right)}{M(P r, P r+1,-P r)}$

Where, $M(a, b, z)$ denotes the Kummer's function.

Figures 1 to 13 show, effects of the governing parameters, comparison of the flow and heat transfer characteristics among the nanofluids with nanoparticles $\mathrm{Cu}, \mathrm{Al}_{2} \mathrm{O}_{3}, \mathrm{CuO}$ and $\mathrm{TiO}_{2}$; and (C) CNCS, Mekelle University 
base fluids water and ethylene glycol with Prandtl numbers $\operatorname{Pr}=6.58$ and 29.86, respectively.

Figure 1 shows the influence of magnetic field parameter $M$ on the velocity distribution $f^{\prime}(\eta)$ for different types of nanoparticles with water as base fluid. The presence of transverse magnetic field sets Lorentz force effects, which results in the retarding effect on the velocity field. As the values of magnetic parameter $M$ increases, the retarding force increases and consequently the velocity decreases. It is also noted that the boundary layer thickness reduces as magnetic parameter $\mathrm{M}$ increases, and the $\mathrm{Cu}$-water has higher value of velocity distribution than $\mathrm{TiO}_{2}$.

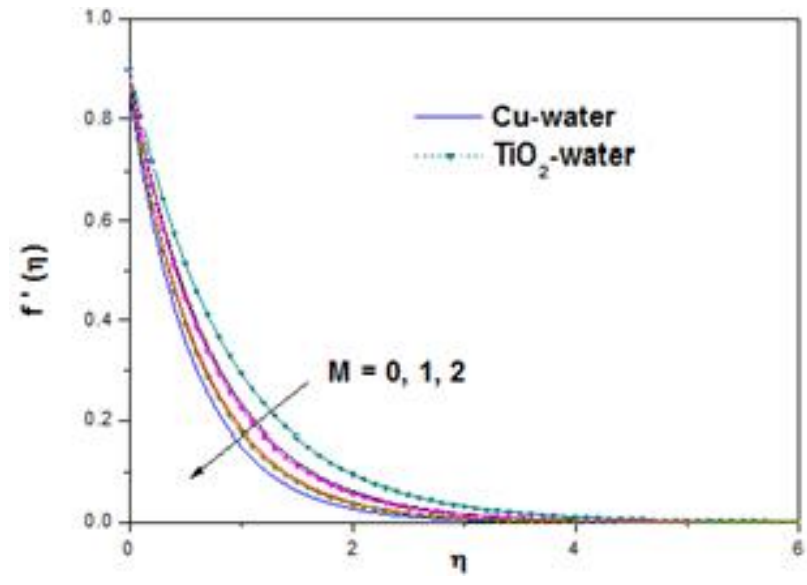

Figure 1. Effects of $\mathrm{M}$ on the velocity distribution $\mathrm{f}^{\prime}(\eta)$ for $\mathrm{Cu}$ - water and $\mathrm{TiO}_{2}-$ water nanofluids, when $\phi=0.2, \mathrm{~K}_{1}=0.3, \gamma=0.1, \mathrm{~h}_{\mathrm{c}}=1$.

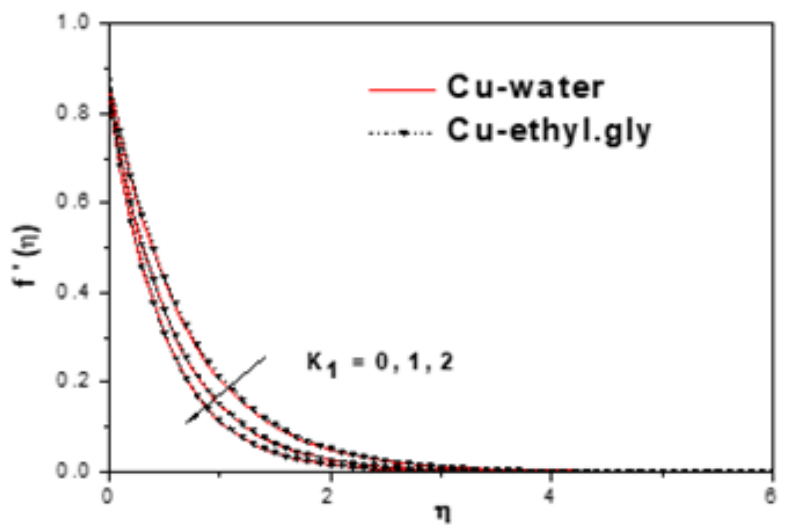

Figure 2. Effects of $\boldsymbol{K}_{\mathbf{1}}$ on the velocity distribution $\boldsymbol{f}^{\prime}(\boldsymbol{\eta})$ for $\boldsymbol{C u}$ - water and $\boldsymbol{C u}$-ethylene glycol nanofluids, when $\phi=0.2, M=1, \gamma=0.1, h_{c}=1$.

Figures 2 and 3 show effects of porosity parameter $K_{1}$ and velocity slip parameter $\gamma$ on the velocity distribution for different types of nanofluids. It is seen that the velocity distribution decreases with increase in the values $K_{1}$ and $\gamma$. The velocity of the nanofluid with ethylene glycol (C) CNCS, Mekelle University

ISSN: $2220-184 X$ 
base fluid is slightly higher than with water for both the $\mathrm{Cu}$ and $\mathrm{Al}_{2} \mathrm{O}_{3}$ nanoparticles.

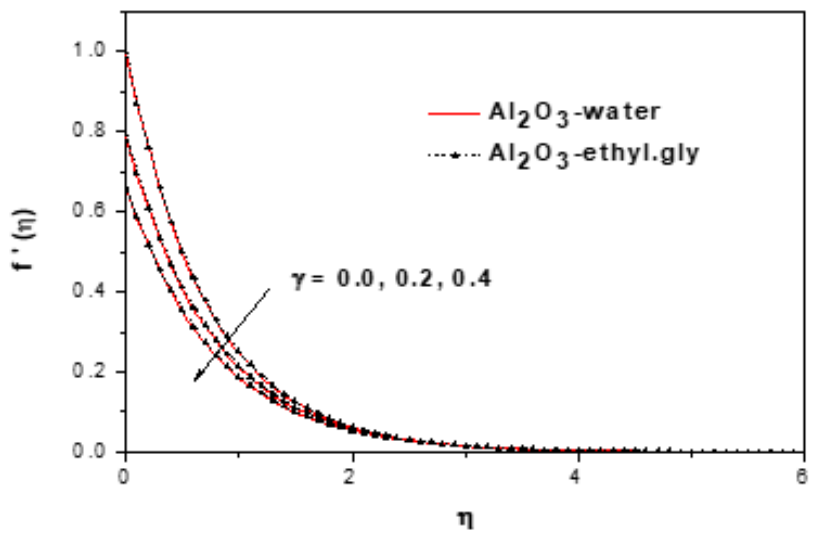

Figure 3. Effects of $\gamma$ on the velocity distribution $f^{\prime}(\eta)$ for $\mathrm{Al}_{2} \mathrm{O}_{3}$ - water and $\mathrm{Al}_{2} \mathrm{O}_{3}$-ethylene glycol nanofluids, when $\phi=0.2, M=1, K_{1}=0.3, h_{c}=1$.

Moreover, from figures 1 to 3 , it can be seen that the velocity boundary layer thickness decreases with an increase in the values of $M, K_{1}$ and $\gamma$. Besides, it can be observed that in the presence of the partial slip, the velocity of the fluid near the surface is lesser than the velocity of the stretching sheet.

Variation of temperature distribution among the nanofluids with $\mathrm{Cu}, \mathrm{Al}_{2} \mathrm{O}_{3}, \mathrm{CuO}$ and $\mathrm{TiO}_{2}$ nanoparticles in water base fluid as shown in figure 4 indicates that the temperature profile is influenced by the type of the nanoparticles. It is also observed that the thermal boundary layer is higher for $\mathrm{Cu}$ compared to $\mathrm{CuO}, \mathrm{A1}_{2} \mathrm{O}_{3}$ and $\mathrm{TiO}_{2}$ nanoparticles in water base fluid. This is because of the high thermal diffusivity attributed to its high thermal conductivity of $\mathrm{Cu}$ compared to the others.

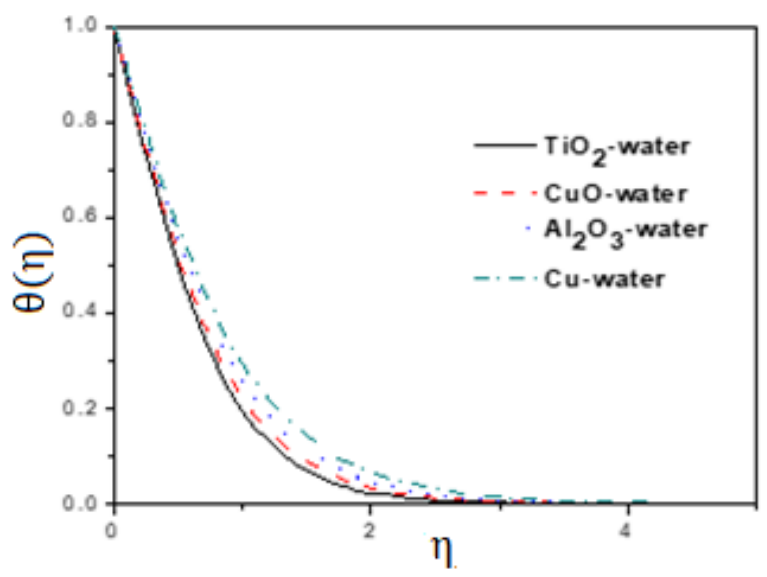

Figure 4. Comparison of temperature distribution $\theta(\eta)$ in $\mathrm{Cu}, \mathrm{CuO}, \mathrm{Al}_{2} \mathrm{O}_{3}$ and $\mathrm{TiO}_{2}$ nanopartticles wtih water base fluid, when $\phi=0.2, \mathrm{M}=1, \mathrm{~K}_{1}=0.3, \gamma=0.1, \mathrm{~h}_{\mathrm{c}}=1$. 
Figures 5 to 9 shows the effects of solid volume fraction $\phi$, magnetic parameter $M$, porosity parameter $K_{1}$, velocity slip parameter $\gamma$ and convective parameter $h_{c}$ on the temperature distribution $\theta(\eta)$ for different types of nanofluids. Figure 5 show effects of $\phi$ on $\theta(\eta)$ for the $\mathrm{Cu}-$ water and $\mathrm{CuO}$-water nanofluids. It illustrates that, the temperature distribution increases with $\phi$. Also, the temperature is lower for a regular fluid ( $\phi=0$, water) compared to the nanofluids $(\phi \neq$ 0), $\mathrm{Cu}$-water and $\mathrm{CuO}$-water. Moreover, the $\mathrm{Cu}$-water has a thicker thermal boundary layer than $\mathrm{CuO}$-water.

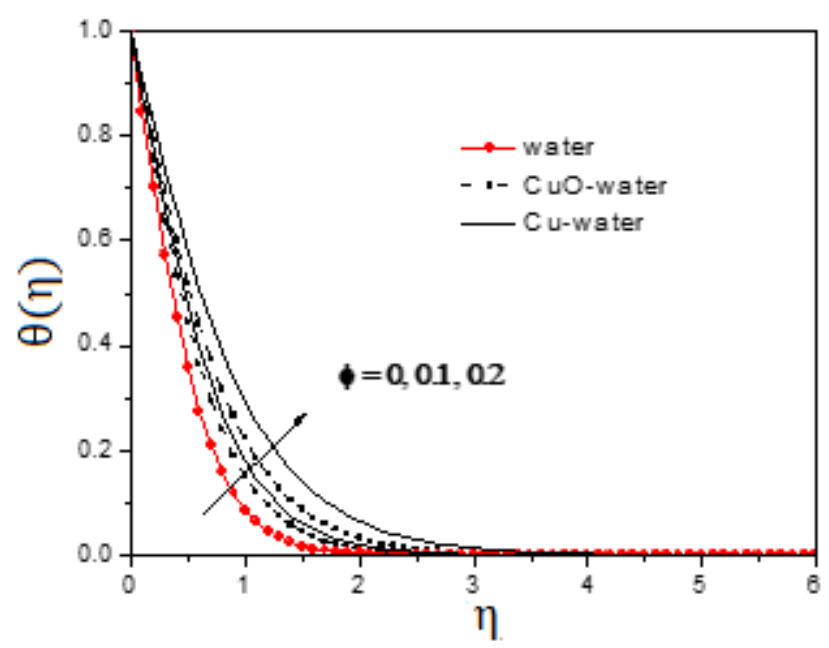

Figure 5. Effects of $\boldsymbol{\phi}$ on temperature distribution $\boldsymbol{\theta}(\boldsymbol{\eta})$ for $\boldsymbol{C u}$-water and $\boldsymbol{C} \boldsymbol{u} \boldsymbol{O}$-water nanofluids, when, $M=1, K_{1}=0.3, \gamma=0.1, h_{c}=1$.

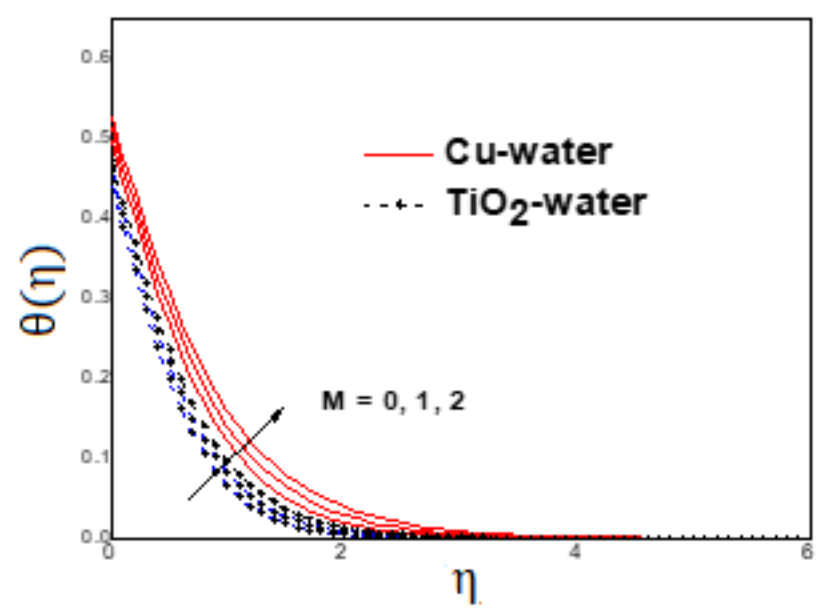

Figure 6. Effects of $\mathrm{M}$ on temperature distribution $\theta(\eta)$ for $\mathrm{Cu}$-water and $\mathrm{TiO}_{2}-$ water nanofluids, when $\phi=0.2, K_{1}=0.3, \gamma=0.1, h_{c}=1$.

Figure 6 describes the effects of $\mathrm{M}$ on temperature profile. It is noticed that the temperature increases with increasing values of $\mathrm{M}$. Figures 7 and 8 represent the effects of porosity parameter (C) CNCS, Mekelle University

ISSN: 2220-184X 
and velocity slip parameter on the temperature profile for different nanoparticles and base fluids. The graphs show that, the temperature at a fixed value of $\eta$ is observed to decrease with an increase in the Prandtl number of the base fluid. This is due to the fact that a higher Prandtl number fluid has relatively low thermal diffusivity, which reduces conduction and thereby the thermal boundary-layer thickness and temperature of the nanofluid decreases.

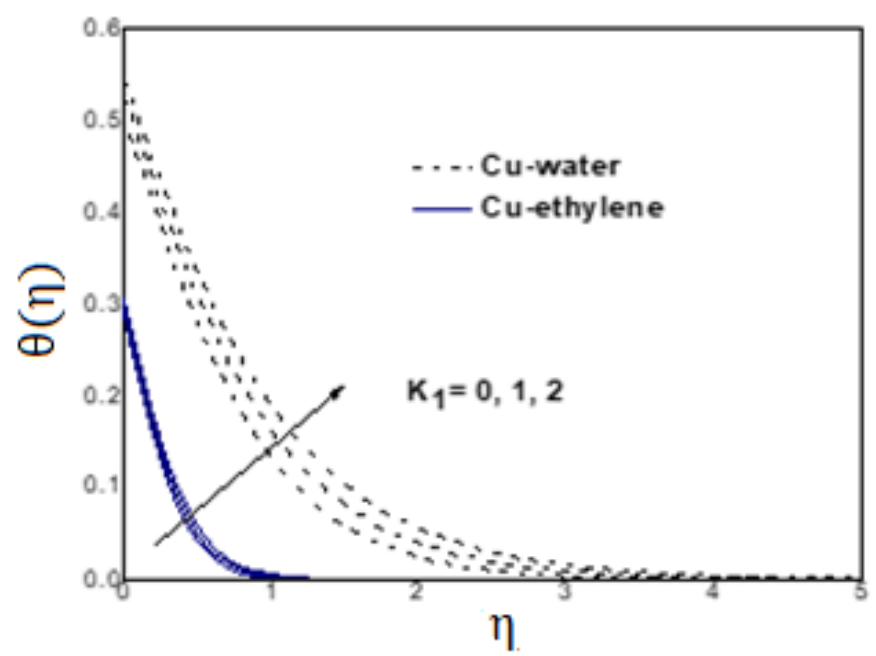

Figure 7. Effects of $K_{1}$ on temperature distribution $\theta(\eta)$ for $\mathrm{Cu}$-water and $\mathrm{Cu}$-ethylene glycol nanofluids, when $\mathrm{M}=1, \phi=0.2, \gamma=0.1, \mathrm{~h}_{\mathrm{c}}=1$.

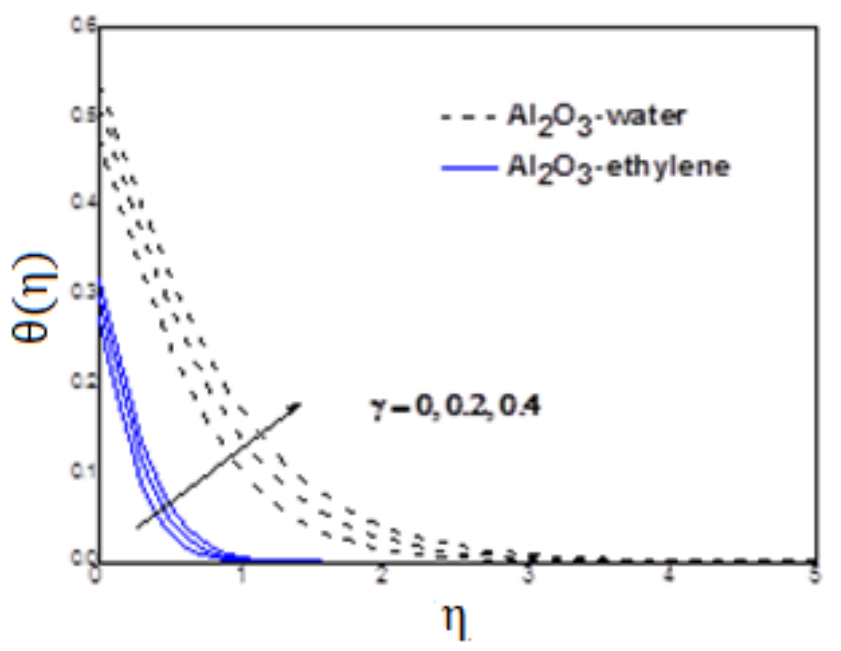

Figure 8. Effects of $\gamma$ on temperature distribution $\theta(\eta)$ for $\mathrm{Al}_{2} \mathrm{O}_{3}$-water and $\mathrm{Al}_{2} \mathrm{O}_{3}$-ethylene glycol nanofluids, when $\mathrm{M}=1, \phi=0.2, \mathrm{~K}_{1}=0.3, \mathrm{~h}_{\mathrm{c}}=1$.

It is seen in figures 7 and 8 that the temperature distribution increases with porosity parameter and velocity slip parameter, respectively. The $\mathrm{Cu}$-water nanofluids has the highest 
temperature among the considered nanofluids, this is because of the high thermal conductivity of $\mathrm{Cu}$ compared to the others.

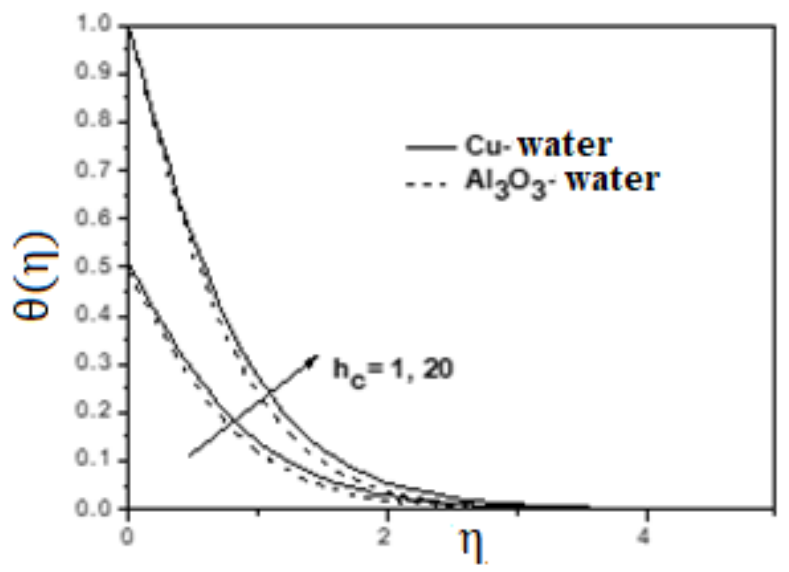

Figure 9. Effects of $h_{c}$ on temperature distribution $\theta(\eta)$ for $\mathrm{Cu}$-water and $\mathrm{Al}_{2} \mathrm{O}_{3}$-ethylene glycol nanofluids, when $\mathrm{M}=1, \phi=0.2, \mathrm{~K}_{1}=0.3, \gamma=0.1$.

In figures 5 to 9 , temperature of the nanofluids is found to be an increasing function of $\phi$, $\mathrm{M}, K_{1}, \gamma$ and $h_{c}$. The temperature boundary layer thickness also increases with $\phi, \mathrm{M}, K_{1}, \gamma$ and $h_{c}$.

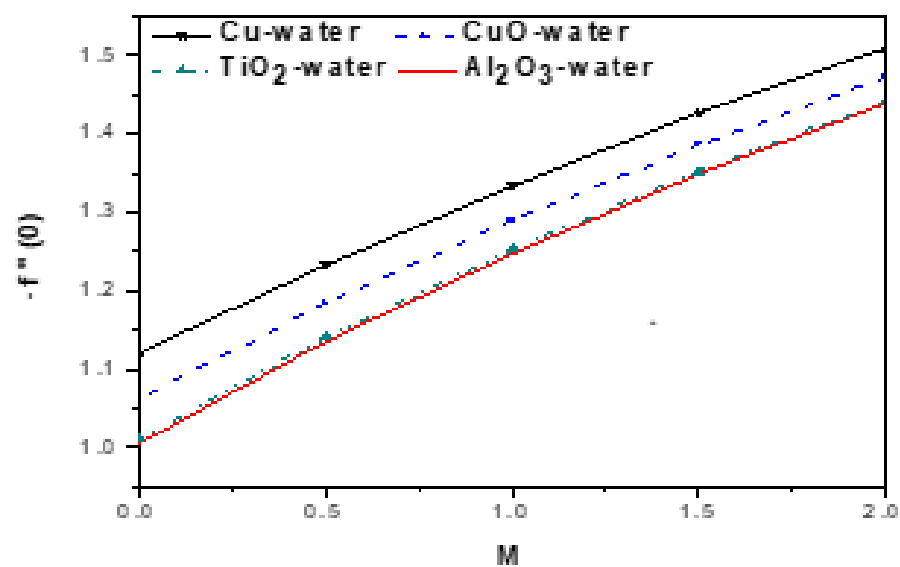

Figure 10. Variation of skin friction coefficient $-\mathrm{f}^{\prime \prime}(0)$ with $\mathrm{M}$ in the $\mathrm{Cu}, \mathrm{CuO}, \mathrm{Al}_{2} \mathrm{O}_{3}$ and $\mathrm{TiO}_{2}$ nanopartticles wtih water base fluid, when $\mathrm{M}=1, \phi=0.2, \mathrm{~K}_{1}=0.3, \gamma=0.1, \mathrm{~h}_{\mathrm{c}}=1$.

Variation of the dimensionless skin friction coefficient $-f^{\prime \prime}(0)$ with the magnetic parameter $M$, porosity parameter $K_{1}$ and velocity slip parameter $\gamma$ for different nanofluids are presented in figures 10 and 11. It is seen that, the skin friction coefficient increases (in absolute value) with $M$ and $K_{1}$, while it decreases with an increase in $\gamma$. Moreover, the highest sheet surface 
stress occur for the $\mathrm{Cu}$-water nanofluid in the no-slip velocity condition $(\gamma=0)$ compared to the $\mathrm{Al}_{2} \mathrm{O}_{3}$-water, $\mathrm{CuO}$ - water and $\mathrm{TiO}_{2}$-water nanofluids.

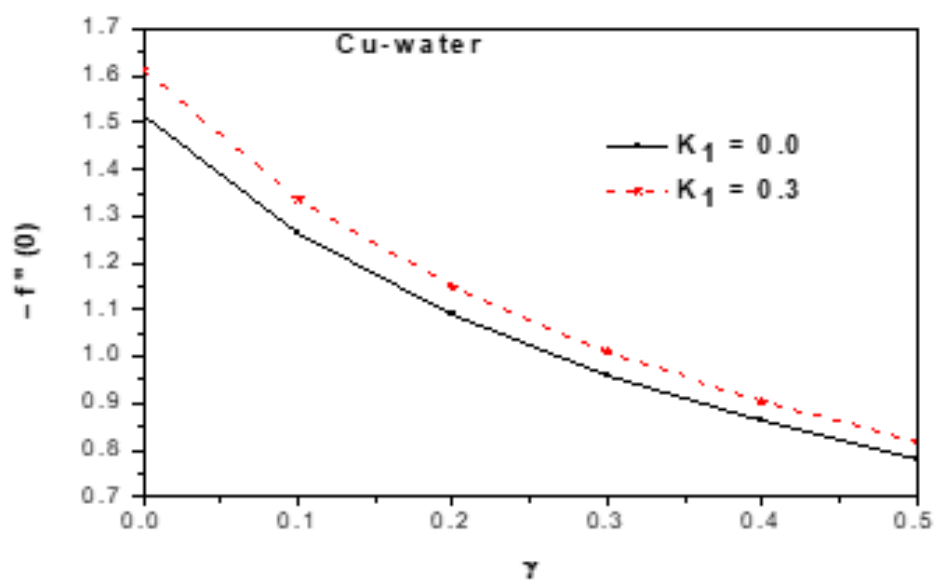

Figure 11. Variation of skin friction coefficient $-\mathrm{f}^{\prime \prime}(0)$ with $\gamma$ for different values of $\mathrm{K}_{1}$ in the $\mathrm{Cu}$-water nanofluid, when $\mathrm{M}=1, \phi=0.2, \mathrm{~h}_{\mathrm{c}}=1$.

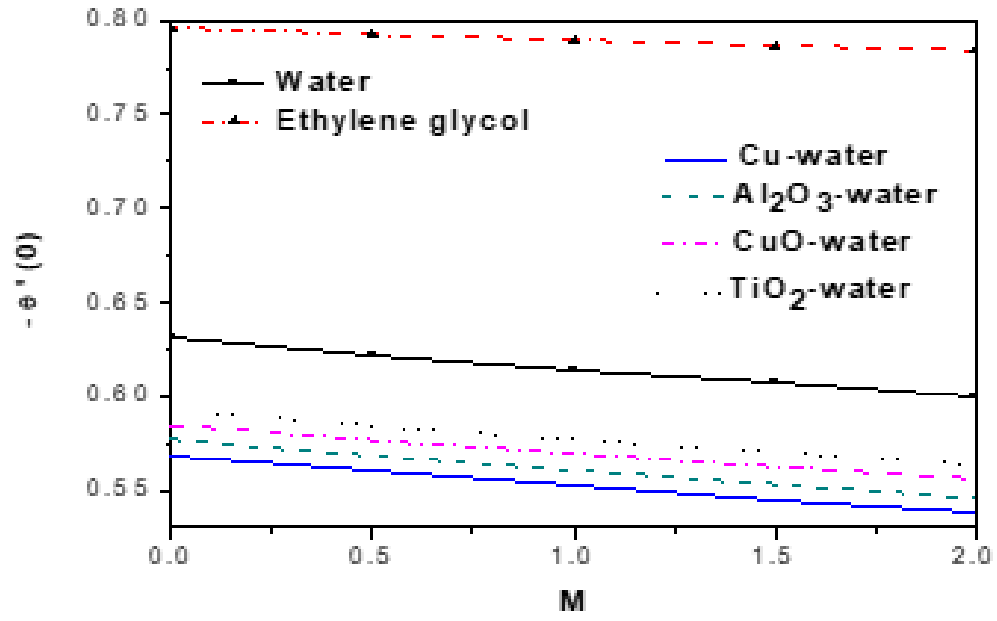

Figure 12. Variation of local Nusselt number $-\theta^{\prime}(0)$ with $\mathrm{M}$ in water, ethylene glycol, $\mathrm{Cu}-$ water, $\mathrm{CuO}$ - water, $\mathrm{Al}_{2} \mathrm{O}_{3}$ - water and $\mathrm{TiO}_{2}$ - water, when $\phi=0.2, \mathrm{~K}_{1}=0.3, \gamma=$ $0.1, \mathrm{~h}_{\mathrm{c}}=1$.

The variation of local Nusselt number $-\theta^{\prime}(0)$ with the magnetic parameter $M$ for different values of the nanofluids and common fluids is shown in figure 12. Since, Prandtl number signifies the relative influence of momentum diffusion to thermal diffusion. When Pr equals unity both the momentum and the temperature diffuse at the same rate. In the present analysis we have chosen the values of Prandtl number those represent the diffusing base fluids of common interest. The 
Values of Pr for water and ethylene glycol are 6.58 and 29.86 respectively. As the Prandtl number increases, the rate of heat transfer increase. It is seen that the higher local Nusselt number occurs in the pure base fluid (i.e, $\phi=0$ ) for water and ethylene glycol. In our case, the fluid with higher Prandtl number, ethylene glycol has the highest rate of heat transfer in the surface compared to water and the nanofluids. Moreover, the Nusselt number is a decreasing function of $M$. Furthermore, the $\mathrm{TiO}_{2}$-water has the highest rate of heat transfer on the surface of the sheet compared to $\mathrm{Cu}$ - water, $\mathrm{Al}_{2} \mathrm{O}_{3}$-water and $\mathrm{CuO}$-water nanofluids.

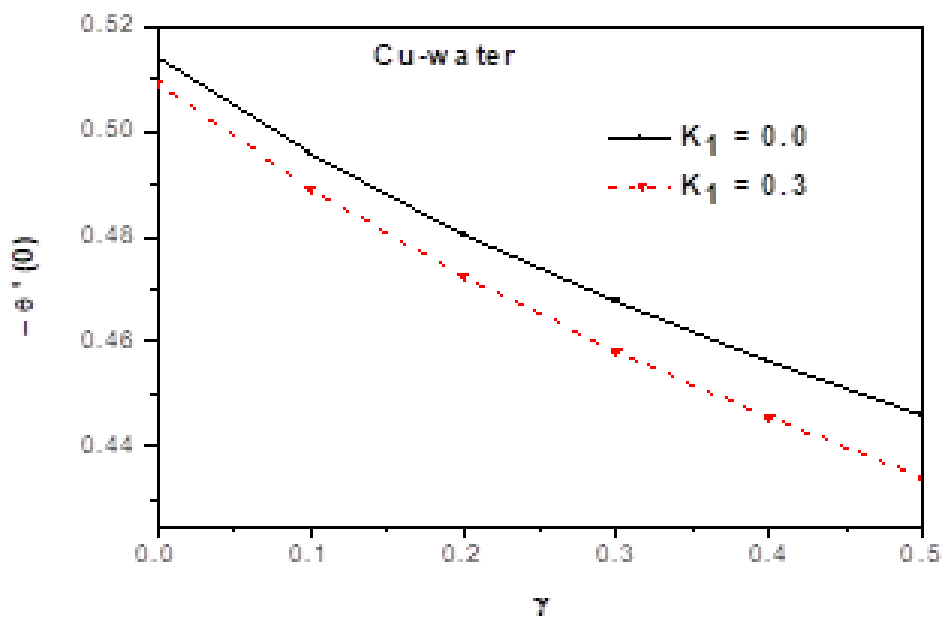

Figure 13. Variation of local Nusselt number $-\theta^{\prime}(0)$ with $\gamma$ for different values of $\mathrm{K}_{1}$ in the $\mathrm{Cu}-$ water nanfofluid, when $\mathrm{M}=1, \phi=0.2,=, \mathrm{h}_{\mathrm{c}}=1$.

Figure 13, illustrates the combined effect of the velocity partial slip parameter $\gamma$ and porosity parameter $K_{1}$ on the local Nusselt number $-\theta^{\prime}(0)$ for the $C u$-water nanofluid. It is observed that the local Nusselt number is a decreasing function of $\gamma$ and $K_{1}$. Hence, to achieve a high rate of heat transfer, less slip on the fluid-solid interface is desired.

\section{CONCLUSION}

In this paper, the MHD partial slip flow and heat transfer of nanofluids through a porous medium over a stretching sheet with convective boundary condition is investigated numerically for the $\mathrm{Cu}, \mathrm{Al}_{2} \mathrm{O}_{3}, \mathrm{CuO}$, and $\mathrm{TiO}_{2}$ nanoparticles with base fluids water and ethylene glycol. The governing nonlinear partial differential equations were transformed into ordinary differential equations using the similarity approach and solved numerically using the fourth order Runge-Kutta method with shooting techniques. Our numerical results revealed, among others, the following. 
The velocity distribution and the thickness of the velocity boundary layer decreases with increasing magnetic parameter $\mathrm{M}$, porosity parameter $K_{1}$ and velocity slip parameter $\gamma$. The $\mathrm{TiO}_{2}$-ethylene glycol has thicker velocity boundary layer compared to the other nanofluids.

$>$ An increment in the solid volume fraction $\phi$, magnetic parameter $M$, porosity parameter $K_{1}$, velocity partial slip parameter $\gamma$ and convective parameter $h_{c}$ yields an increment in the nanofluids temperature, this leads to a decrease in the heat transfer rates. The $\mathrm{Cu}$ - water has the highest thermal boundary layer thickness compared to the other nanofluids, this is due to the high thermal conductivity of $\mathrm{Cu}$.

The skin friction coefficient increases with increasing the values of the magnetic parameter $M$, porosity parameter $K_{1}$ and velocity slip parameter. The $\mathrm{Cu}-$ water show the highest drag effect to the flow compared to the other nanofluids.

$>$ The heat transfer rate at the plate surface decreases with increasing $\phi, M, K_{1}$ and $\gamma$. Highest rate of heat transfer occurs in the ethylene glycol and the nanofluid with $\mathrm{TiO}_{2}$ nanoparticle has the highest cooling performance than the other nanoparticles $\left(\mathrm{Cu}, \mathrm{Al}_{2} \mathrm{O}_{3}\right.$ and $\mathrm{CuO})$.

A comparison to validate the accuracy of the present results with previously published papers has shown an excellent agreement.

\section{ACKNOWLEDGEMNETS}

I would like to thank Prof. Bandari Shankar, Osmania University, Hyderabad, India; and Dr. Gebregiorgis Abraha, Mekelle University, Mekelle, Tigray, Ethiopia for their continuous professional support and advice during the preparation of this article.

\section{REFERENCE}

Abel, M. S., Nandeppanavar, M. M \& Malipatil, S. B. 2010a. Heat transfer in a second grade fluid through a porous medium from a permeable stretching sheet with non-uniform heat source/sink. International Journal Heat Mass Transfer, 53(9-10); 1788-1795.

Abel, M. S., Nandeppanavar, M. M \& Malkhed, M. B. 2010b. Hydromagnetic boundary layer flow and heat transfer in a fluid over a continuously moving permeable stretching surface with non-uniform heat source/sink embedded in fluid saturated porous medium. Chemical 
Engineering Communications. 197(5): 633-655.

Abel, M. S., Mahesha, N \& Malipatel, S. B. 2010c. Heat transfer due to MHD slip flow of a second grade liquid over a stretching sheet through a porous medium with non-uniform heat source/sink. Chemical Engineering Communications. 198(2):191-213.

Abramowitz, M \& Stegun, I. A. 1965. Handbook of Mathematical Functions. Dover Publications, ISBN-10: 0486612724, ISBN-10:0486612724, 1046p.

Ahmad, S., Rohni, A. M \& Pop, I. 2011. Blasius and Sakiadis problems in nanofluids. Acta Mechanica, 218(3): 195-204.

Anuar Ishak. 2010. Unsteady MHD flow and heat transfer over a stretching plate. Journal of Applied Sciences, 10(18): 2127-2131.

Attia, H. A., Mostafa, A. M. Abdeen \& Karem Mahmoud Ewis. 2012. Stagnation Point Flow through a Porous Medium towards a Radially Stretching Sheet in the Presence of Uniform Suction or Injection and Heat Generation. J. Fluids Engineering, 134(8): 081202-1-5.

Attia, H. A. 2007. On the effectiveness of porosity on stagnation point flow towards a stretching surface with heat generation, Computational Materials Science, 38(4): 741-745.

Aziz, A. 2009. Similarity solution for laminar thermal boundary layer over a flat plate with a convective surface boundary condition. Communication in Nonlinear Science and Numerical Simulation, 14(4): 1064-1068.

Aziz, A. 2010. Hydrodynamic and thermal slip flow boundary layer over a flat plate with constant heat flux boundary condition. Communication in Nonlinear Science and Numerical Simulation, 15(3): 573-580.

Beg, A., Bakier, A.Y \& Prasad, V. R. 2009. Numerical study of free convection magnetohydrodynamic heat and mass transfer from a stretching surface to a saturated porous medium with Soret and Dufour effects. Computer Mater. Sci. 46: 57-65.

Buongiorno, J \& Hu, W. 2005. Nanofluid coolants for advanced nuclear power plants. Proc. of ICAPP 05: May 2005, Seoul. Sydney: Curran Associates, Inc, pp.15-19.

Buongiorno, J. 2006. Convective transport in nanofluids, ASME J Heat Trans, 128: 240- 250.

Damseh, R. A \& Duwairi, H. M. 2008. Thermophoresis particle deposition: Natural convection interaction from vertical permeable surfaces embedded in a porous medium. J. Porous Media, 12: 79-88. 
Dash, S., Dash, G. C \& Misra, D. P. 2008. The MHD flow through a porous medium past a stretched vertical permeable surface in the presence of heat source/sink and a chemical reaction. Proc. National Academy of Sciences India, A 78(1): 49-55.

Fang, T., Yao, S., Zhang, J \& Aziz, A. 2010. Viscous flow over a shrinking sheet with a second order slip flow model. Commun. Nonlinear Sci. and Num. Simul., 15(7): 1831-1842.

Fang, T., Zhang, J \& Yao, S. 2009. Slip MHD viscous flow over a stretching sheet: an exact solution. Commun. Nonlinear Sci. Numer. Simul., 14(11): 3731-3737.

Fang, T., Zhang, J \& Yao, S. 2010. Slip magnetohydrodynamic viscous flow over a permeable shrinking sheet. Chin. Phys. Lett., 27(12): 124702.

Ganesan, P \& Palani, G. 2004. Finite difference analysis of unsteady natural convection MHD flow past an inclined plate with variable surface heat and mass flux, Int. J. Heat and Mass Transfer, 47(19-20): 4449-4457.

Grubka, L. J \& Bobba, K. M. 1985. Heat transfer characteristics of a continuous stretching surface with variable temperature. J. Heat Transfer, 107(1): 248-250.

Hamada, M.A.A., Pop, I \& Ismail, A.I. Md. 2011. Magnetic field effects on free convection flow of a nanofluid past a vertical semi- infinite flat plate. Nonlinear Analysis Real World Applications, 12: 1338-1346.

Hamad, M. A. A \& Pop, I. 2011. Scaling transformations for boundary layer flow near the stagnation point on a heated permeable stretching surface in a porous medium saturated with a nanofluid and heat generation/absorption effects. Transp. Porous Media, 87: 25-39.

Hayat, T, Hussain, Q \& Javed T. 2009. The modified decomposition method and pade approximation for the MHD flow over a non-linear stretching sheet. Nonlinear Analysis Real World Applications, 10(2): 966-973.

Ishak, A. 2010. Similarity solution for flow and heat transfer over a permeable surface with convective boundary condition. Appl. Math. Comput., 217: 837-842.

Ishak, A., Yacob, A \& Bachok, N. 1985. Radiation effect on the thermal boundary layer flow over a moving plate with convective condition. Meccanica, 46: 795-801.

Jafar, K., Nazar, R., Ishak, A \& Pop, I. 2011. MHD Flow and Heat Transfer Over stretching/shrinking sheets with external Magnetic field, viscous dissipation and Joule Effects. Can. J. Chem. Eng, 90(5): 1-11 (DOI: 10.1002/cjce.20609). . 
Kang, H.U., Kim, S.H \& Oh, J.M. 2006. Estimation of thermal conductivity of nanofluid using experimental effective particle volume. Exp. Heat Transfer, 19(3): 181-191.

Khan, W. A \& Pop I. 2011. Free convection boundary layer flow past a horizontal flat plate embedded in a porous medium filled with a nanofluid. J. Heat Transfer, 133: 9.

Magyari, E \& Postelnicu, A. 2011. Double-diffusive natural convection flows with thermosolutal symmetry in porous media in the presence of the Soret-Dufour effects. Transp. Porous Media, 88: 147-167.

Makinde, O. D. 2012. Entropy analysis for MHD boundary layer flow and heat transfer over a flat plate with a convective surface boundary condition, Int. J. Exergy, 10(2): 142-154.

Makinde, O.D \& Aziz A. 2010. MHD mixed convection from a vertical plate embedded in a porous medium with a convective boundary condition. Int. J. Thermal Sci., 49: 1813-1820.

Makinde, O.D \& Olanrewaju, P. 2010. Buoyancy effect on thermal boundary layer over a vertical plate with a convective surface boundary condition. ASME J. Fluids Eng.,132(044502):14.

Markin, H \& Pop, I. 2011. The forced convection flow of a uniform stream over a flat surface with a convective surface boundary condition. Commun. Nonlinear Sci. Numer.Simul., 16: 3602-3609.

Masuda, H., Ebata, A., Teramae, K \& Hishinuma, N. 1993. Alteration of thermal conductivity and viscosity of liquid by dispersing ultra-fine particles. Netsu Bussei, 7: 227-233.

Oztop, H.F \& Abu-Nada, E. 2008. Numerical study of natural convection in partially heated rectangular enclosures filled with nanofluids. Int. J. Heat Fluid Flow, 29: 1326-1336.

Rudyak, V.Y., Belkin, A.A.and Tomilina, E.A. 2010. On the thermal conductivity of nanofluids. Tech. Phys. Lett., 36(7): 660-662.

Sarit, K., Das S., Wenhua, Y \& Pradeep, T. 2007. Nanofluids Science and Technology. $1^{\text {st }}$ edition, Honboken, NJ, John Wiley \& Sons, Inc.

Tiwari, R.K \& Das, M.N. 2007. Heat transfer augmentation in a two sided lid $\ddot{i} i^{1 / 2}$ driven differentially heated square cavity utilizing nanofluids. International J. Heat and Mass Transfer, 50: 2002-2018.

Turkyilmazoglua, M \& Popb, I. 2013. Heat and mass transfer of unsteady natural convection flow of some nanofluids past a vertical infinite flat plate with radiation effect. Int. J. Heat and 
Mass Transfer, 59: 167-171.

Velagapudi, V., Konijeti, R.K \& Aduru, C.S.K. 2008. Empirical correlation to predict thermophysical and heat transfer characteristics of nanofluids. Therm. Sci., 12(2): 27-37.

Wang, C. Y. 2009. Analysis of viscous flow due to a stretching sheet with surface slip and suction. Nonlinear Analysis Real World Appl., 10(1): 375-380.

Wubshet, I \& Shankar, B. 2012. Boundary-Layer Flow and Heat Transfer of Nanofluid Over a Vertical Plate With Convective Surface Boundary Condition. ASME J. Fluids Eng., 134(8): 081203.

Wubshet, I., Shankar, B \& Mahantesh, M. N. 2013. MHD stagnation point flow and heat transfer due to nanofluid towards a stretching sheet. Int. J. Heat and Mass Transfer, 56(1-2): 1-9.

Xua, H., Pop, I \& Youc, X. 2013. Flow and heat transfer in a nano- liquid film over an unsteady stretching surface. Int. J. Heat and Mass Transfer, 60: 646-652.

Yacob, A., Ishak, A., Pop, I \& Vajavelu, K. 2011. Boundary layer flow past a stretching/Shrinking surface beneath an external uniform shear flow with convective surface boundary condition in a nanofluid. Nanoscale Res. Lett., 6(314): 1-7.

Yao, S., Fang, T \& Zhong, Y. 2011. Heat transfer of a generalized stretching/shrinking wall problem with convective boundary condition. Commun. Nonlinear Sci. Numer. Simul., 16: $752-760$.

Yu, W., France, D. M., Routbort, J. L \& Choi, S. U. S. 2008. Review and Comparison of Nanofluid Thermal Conductivity and Heat Transfer Enhancements. Heat Transfer Engineering, 29(5): 432-460. 